PROCEEDINGS OF THE

AMERICAN MATHEMATICAL SOCIETY

Volume 133, Number 5, Pages 1515-1525

S 0002-9939(04)07740-8

Article electronically published on December 15, 2004

\title{
ORBITAL INTEGRALS ARE MOTIVIC
}

\author{
THOMAS C. HALES \\ (Communicated by Dan M. Barbasch)
}

\begin{abstract}
This article shows that under general conditions, $p$-adic orbital integrals of definable functions are represented as the trace of a Frobenius operator on a virtual motive. This gives an explicit example of the philosophy of Denef and Loeser, which predicts that all "naturally occurring" $p$-adic integrals are motivic.
\end{abstract}

\section{INTRODUCTION}

Denef and Loeser have introduced a theory of arithmetic motivic integration [3]. In this theory, general families of $p$-adic integrals can be calculated as the trace of a Frobenius operator on virtual motives. This article shows that orbital integrals fit nicely into the general framework of Denef and Loeser. It describes a large class of orbital integrals that can be computed as the trace of a Frobenius operator. Moreover, there is an effective procedure to compute the algebraic varieties that represent the integrals. In this sense, this article gives an algorithm to compute a large class of orbital integrals.

The idea of using double cosets to compute motivic orbital integrals is taken from J. Gordon's recent thesis (and a suggestion of Julee Kim). The thesis proves that under general conditions, the character values of depth zero representations of a $p$-adic group can be represented as virtual motives [10. This note can be viewed as an extension of the methods of that thesis.

\section{Pas's LANGUAGe AND MOtives}

Recall that Pas has defined a first-order language that is based on the theory of valued fields [14]. It is a three-sorted language in the sense of [8]. The structures of the three sorts are a valued field, a residue field, and the additive group of integers (the value group), augmented by $+\infty$. The language has function symbols ord and ac that are interpreted as the valuation and angular component maps on a valued field. The valuation is a map from the valued field to $\mathbb{Z} \cup\{+\infty\}$. The angular component map is a function from the valued field to its residue field.

Received by the editors January 7, 2002 and, in revised form, October 24, 2003.

2000 Mathematics Subject Classification. Primary 22E50.

This research was supported by NSF grant 245332 .

(C)2004 American Mathematical Society Reverts to public domain 28 years from publication 
2.1. Quantifier elimination. Let $\mathcal{K}$ be a set of structures for Pas's language. We do not make any assumptions about the residual characteristic of the fields in $\mathcal{K}$. We do, however, assume that each field is complete and Henselian. In fact, the only cases of interest to us are locally compact nonarchimedean fields (in brief, $p$-adic fields). For example, we could take $\mathcal{K}$ to be structures corresponding to the set of $p$-adic fields $\mathbb{Q}_{p}$, or the set of fields $\mathbb{F}_{p}((t))$, or the set of all $p$-adic fields. We write $\theta^{k}$ for an interpretation of $\theta$ in the structure $k$. If $S$ is a finite set of prime numbers, let $\mathcal{K}_{S}$ be the set of $k \in \mathcal{K}$ for which the residual characteristic of $k$ is not in $S$.

Let $\theta(m)$ be a formula in Pas's language. Assume that $\theta$ has no free variables of the valued field sort or of the residue field sort. Assume that its free variables of the additive sort are contained in $m=\left(m_{1}, \ldots, m_{\ell}\right)$.

Suppose that there exists a finite set $S$ of prime numbers satisfying the following condition:

Condition 1. For every $k \in \mathcal{K}_{S}$,

$$
\left\{m \in \mathbb{Z}^{\ell}: \theta^{k}(m)\right\}
$$

is a bounded subset of $\mathbb{Z}^{\ell}$.

Theorem 2. Under the stated conditions, there exists a finite set $S^{\prime}$ of prime numbers and a bounded subset $C \subset \mathbb{Z}^{\ell}$ such that for every $k \in \mathcal{K}_{S^{\prime}}$ we have

$$
\left\{\left(m_{1}, \ldots, m_{\ell}\right) \in \mathbb{Z}^{\ell}: \theta^{k}\left(m_{1}, \ldots, m_{\ell}\right)\right\} \subset C .
$$

In other words, by throwing away finitely many primes, the bound on the subset in Condition 1 can be made independent of the structure.

Proof. Apply Pas's quantifier elimination on quantifiers of the valued field sort in the formula $\theta$. We obtain an equivalent formula that contains no variables of the valued field sort. This formula is uniform in the sense that it is independent of the structure $k \in \mathcal{K}$. Pas assumes that the residual characteristic is zero. We achieve equivalent results by throwing out finitely many residual characteristics.

The remaining terms of the valued field sort are constants that are definable in Pas's language without quantifiers. Such a constant must be a rational number. Equalities in the valued field $a=b$ can be replaced with an equivalent statement with the angular component map: $\operatorname{ac}(a-b)=0$. The infinite valuation condition $\operatorname{ord}(a)=+\infty$ can be replaced with $\operatorname{ac}(a)=0$. Excluding finitely many primes, each rational number appearing in the formula can be assumed to be 0 or a unit; that is, it is an integer in each structure. If $a \neq 0$, the condition ord $(a)=m$ is then equivalent to the formula $m=0$, and if $a=0$ it is false (because we have already treated $\operatorname{ord}(a)=+\infty)$. Similarly, the formula $\operatorname{ac}(a)=\xi$ is equivalent to $a^{\prime}=\xi$, where $a^{\prime}$ is the element in the residue field that is the reduction of the non-zero rational number $a$. In this way, we eliminate all terms of the valued-field sort, and all occurrences of the function symbols ac and ord.

Writing the formula in disjunctive normal form we find that $\theta$ is equivalent to

$$
\bigvee_{i}\left(\psi_{i} \wedge L_{i}\right),
$$

where $\psi_{i}$ is a formula with no free variables and containing only constants and terms of the residue field sort, and $L_{i}$ is a formula with free variables limited to $m$ and whose constants and terms are of the value group sort. We may assume 
that $+\infty$ has been eliminated from the language, so that each $L_{i}$ is a conventional Presburger formula 8 .

We partition the finite set of indices $i$ into two sets $B$ and $\bar{B}$, where $B$ is the set of indices for which $L_{i}$ is a bounded subset of $\mathbb{Z}^{\ell}$ and $\bar{B}$ the set of indices for which $L_{i}$ is unbounded. We can find a set $C \subset \mathbb{Z}^{\ell}$ that contains

$$
\bigcup_{i \in B}\left\{m: L_{i}(m)\right\} \text {. }
$$

Note that $C$ is independent of the structure.

Let $S^{\prime}$ be the union of $S$ with the set of primes that were excluded by Pas's quantifier elimination, together with the primes that were excluded to make the rational constants in the valued field all units (or 0 ).

If $k \in \mathcal{K}_{S^{\prime}}$, then $\theta$ is equivalent to Formula (1) in the interpretation $k$. By hypothesis, the set of solutions is bounded, which implies that each $\psi_{i}^{k}$, for $i \in \bar{B}$, is false. Hence all the solutions lie in $C$, as desired.

2.2. A ring of virtual motives. A ring $K_{0}^{m o t}\left(\operatorname{Var}_{k}\right)\left[\mathbb{L}^{-1}\right] \otimes \mathbb{Q}$ is constructed by Denef and Loeser in [4] and [5]. We briefly recall its definition, and refer the reader to [4] and [5] for details. First of all, $K_{0}\left(\operatorname{Var}_{k}\right)$ is the Grothendieck ring of varieties over a field $k$ of characteristic zero. It is generated by symbols $[V]$, for every variety $V$ over $k$. We omit the relations. Let $\mathbb{L}=\left[\mathbb{A}_{k}^{1}\right]$ be the class of the affine line. $K_{0}^{m o t}\left(\operatorname{Var}_{k}\right)$ is a quotient of $K_{0}\left(\operatorname{Var}_{k}\right)$ that is obtained by killing all L-torsion and by identifying $[V]$ and $[W]$ whenever $[V]$ and $[W]$ are nonsingular projective varieties that become equal in the category of Chow motives. The ring $K_{0}^{m o t}\left(\operatorname{Var}_{k}\right)\left[\mathbb{L}^{-1}\right] \otimes \mathbb{Q}$ is then defined by inverting $\mathbb{L}$ and tensoring with $\mathbb{Q}$. For the purposes of this paper, a virtual motive is an element of the ring $K_{0}^{m o t}\left(\operatorname{Var}_{k}\right)\left[\mathbb{L}^{-1}\right] \otimes \mathbb{Q}$. We use [.] both for elements of the Grothendieck ring $K_{0}\left(\operatorname{Var}_{k}\right)$ and for their images in $K_{0}^{m o t}\left(\operatorname{Var}_{k}\right)\left[\mathbb{L}^{-1}\right] \otimes \mathbb{Q}$.

Denef and Loeser also construct a completion of $K_{0}^{m o t}\left(\operatorname{Var}_{k}\right)\left[\mathbb{L}^{-1}\right] \otimes \mathbb{Q}$. In the special setting that we consider, this completion will not be necessary.

If $L$ is a finite extension of $\mathbb{Q}$, and $v$ is a place of $L$, the completion $L_{v}$ at $v$ is (the domain of) a structure for Pas's language. Let $O_{v}$ be the ring of integers of $L_{v}$.

If $v$ is a place of the field $L$, then there is a Frobenius operator Frob $v$ that acts on the $\ell$-adic cohomology groups of $M$. See [3. 3.3]. We write the alternating trace of Frobenius on the cohomology as

$$
\operatorname{trace}\left(\operatorname{Frob}_{v}, M\right) \in \overline{\mathbb{Q}}_{\ell} .
$$

\section{Some VIRTUAL SETS}

We write

$$
\theta\left(x_{1}, \ldots, x_{\ell}, m_{1}, \ldots, m_{\ell^{\prime}}, \xi_{1}, \ldots, \xi_{\ell^{\prime \prime}}\right)
$$

for a formula in Pas's language, where all free variables are among those listed, and the variables are of the valued field sort $x_{i}$, value group sort $m_{i}$, and residue field sort $\xi_{i}$.

3.1. Virtual sets. In many axiomatic developments of set theory (such as the Zermelo-Fraenkel system), the fundamental entities are sets. Classes, if they make an appearance at all, are introduced as a purely syntactic construct. Classes are 
nothing but syntactic sugar. Every well-formed expression involving a class $\{x$ : $\theta(x)\}$ can be rewritten in a more fundamental notation without classes.

We introduce similar syntactic conventions in the first-order language of rings and Pas's language. However, we follow Quine in using the term virtual set, where other authors would use the word class. Thus, if $\theta$ is a formula in the first-order language of rings or in Pas's language, we introduce $\{x: \theta(x)\}$ as a syntactic construct and call it a virtual set. A standard rewriting process can be used to eliminate virtual sets from well-formed expressions. For example, the expression $\left\{x: \theta_{1}(x)\right\} \subset\left\{x: \theta_{2}(x)\right\}$ can be rewritten as the first-order formula $\forall x . \theta_{1}(x) \Rightarrow$ $\theta_{2}(x)$. See [11, where the corresponding notion for formulas in the first-order theory of rings is discussed.

Example 3. A polynomial ideal $\left(f_{1}, \ldots, f_{\ell}\right) \subset \mathbb{Z}\left[x_{1}, \ldots, x_{n}\right]$ determines a virtual set

$$
Y=\left\{x: f_{1}(x)=0 \wedge \cdots \wedge f_{\ell}(x)=0\right\} .
$$

The structure $Y(F)$ of this virtual set in a field $F$ is the zero set of the ideal in $F^{n}$. In particular, $\mathbb{A}^{n}$ may be viewed as the virtual set associated with the zero ideal (0) $\subset \mathbb{Z}\left[x_{1}, \ldots, x_{n}\right]$. More generally, a constructible set determines a virtual set. In this way, various affine varieties such as affine algebraic groups can be treated as virtual sets.

3.2. Characteristic functions. Let $R$ be the free abelian group on the set of virtual subsets of $\mathbb{A}^{n}$. Let $R_{\mathbb{Q}}=R \otimes \mathbb{Q}$. If $A$ is a virtual subset of $\mathbb{A}^{n}$, then we let $\chi_{A}$ be the corresponding element of $R_{\mathbb{Q}}$. We call $\chi_{A}$ the characteristic function of $A$. The space $R_{\mathbb{Q}}$ is a ring under the multiplication $\chi_{A} \cdot \chi_{B}=\chi_{A \cap B}$. If $F$ is a structure for Pas's language, then we have a characteristic function $\chi_{A}^{F}$ on $F^{n}$, which takes the value 1 on the set where $A(F)$ and 0 elsewhere. We extend linearly to obtain a function $f^{F}$ on $F^{n}$ for every $f \in R_{\mathbb{Q}}$.

If $\theta\left(x_{1}, \ldots, x_{\ell}\right)$ is a formula in Pas's language with no free variables of the residue field sort and no free variables of the value field sort, then we can construct the auxiliary formula $\psi_{\theta}$ :

$$
\begin{aligned}
& \exists m . \forall y_{1}, \ldots, y_{\ell}, x_{1}, \ldots, x_{\ell} \\
& \quad\left(\operatorname{ord}\left(y_{i}-x_{i}\right) \geq m, \text { for } i=1, \ldots, \ell\right) \Rightarrow \\
& \quad\left(\theta\left(x_{1}, \ldots, x_{\ell}\right) \Leftrightarrow \theta\left(y_{1}, \ldots, y_{\ell}\right)\right) .
\end{aligned}
$$

The formula $\psi_{\theta}$ states that if $x$ and $y$ are close, then $x$ satisfies $\theta$ exactly when $y$ does. Closeness in the formula $\psi_{\theta}$ is measured by a parameter $m$ which is independent of $x$ and $y$. The formula $\psi_{\theta}$ does not contain any free variables. By quantifier elimination, the auxiliary formula $\psi_{\theta}$ is equivalent to something of the shape of Formula (11) (excluding finitely many primes). There are no free variables of the value group sort. By Presburger quantifier elimination, the formulas $L_{i}$ can be replaced with "true" or "false". Thus, the formula may be further simplified to a formula in the first-order theory of the residue field sort.

Definition 4. We say that a characteristic function $\chi_{A}$ is locally constant if $A=$ $\{x: \theta(x)\}$ for some formula $\theta$, whose auxiliary formula $\psi_{\theta}$ (viewed as a formula in the residue field sort) is true for all finite fields of sufficiently large characteristic.

We define the $\mathbb{Q}$-span of locally constant characteristic functions in $R_{\mathbb{Q}}$ to be the space of locally constant functions on $\mathbb{A}^{n}$. Locally constant functions on $\mathbb{A}^{n}$ form a 
ring. Sometimes we will call them locally constant virtual functions to emphasize that they are not functions in any traditional sense.

Local constancy asserts that the auxiliary formula $\psi_{\theta}^{\mathbb{F}_{q}}$ is true over all finite fields of sufficiently large characteristic. By avoiding finitely many residue characteristics, each locally constant function $f$ on $\mathbb{A}^{n}$ determines a locally constant function $f^{F}$ on $F^{n}$ for $p$-adic fields $F$.

Remark 5. Denef and Loeser have introduced the notion of definable subassignments [3]. Each virtual set $A$ determines a function $K \mapsto A(K[[t]])$ on the class of fields over a given field $k$. This function is a definable subassignment of $\mathcal{L}\left(\mathbb{A}^{n}\right)$. In fact, whatever we describe as a virtual set could be described in equivalent terms with definable subassignments. In the other direction, if $C$ is a definable subassignment of the arc space $\mathcal{L}\left(\mathbb{A}^{n}\right)$, given by a formula $\theta$ in Pas's language, then we can form the virtual set $A=\{x: \theta(x)\}$ and the characteristic function $\chi_{A}$. If $A$ is a virtual set, then its interpretations $A(F)$ are definable sets.

3.3. Lie algebras. Let $\mathfrak{g}$ be a virtual reductive Lie algebra in the sense of [11]; that is, a virtual set in Pas's language whose interpretations in various structures are reductive Lie algebras in the traditional sense. Assume that $\mathfrak{g}$ is the Lie algebra of a virtual reductive Lie group $G$. (Again, this is to be interpreted as a virtual set whose interpretations are reductive algebraic groups.) For example, each split reductive group over $\mathbb{Q}$ gives a virtual reductive group in Pas's language.

We define the space of locally constant virtual functions on $\mathfrak{g}$ to be the ring of locally constant functions on $\mathbb{A}^{n}$ under an identification of $\mathfrak{g}$ with $\mathbb{A}^{n}$ as linear spaces.

If $Y$ is a vector or matrix of variable symbols in Pas's language (say $Y=$ $\left.\left(y_{1}, \ldots, y_{k}\right)\right)$, then we write $\exists Y$ as shorthand for $\exists y_{1} y_{2} \ldots y_{k}$, and similarly for $\forall Y$.

Let $\ell$ be the rank of $\mathfrak{g}$. Let $\operatorname{span}\left(Y, e_{1}, \ldots, e_{\ell}\right)$ be the formula in the first-order language of rings asserting that $Y$ lies in the span of the $e_{i}$. The set of regular elements $\mathfrak{g}^{r e g}$ is defined as a virtual set of elements in $\mathfrak{g}$ :

$$
\left\{X: \exists e_{1}, \ldots, e_{\ell} \forall Y . \quad[X, Y]=0 \Rightarrow \operatorname{span}\left(Y, e_{1}, \ldots, e_{\ell}\right)\right\} .
$$

The set of nilpotent elements in $\mathfrak{g}$ is a subvariety, hence a virtual subset in Pas's language. A regular semisimple element is defined as a regular element that has 0 as the only nilpotent element in the centralizer. This condition is given by a formula, hence regular semisimple elements form a virtual set $\mathfrak{g}^{\text {reg,ss }}$.

In a split reductive algebra over $\mathbb{Q}$, we have a finite list of definable subsets of $\mathfrak{g}$ giving all of the proper parabolic subgroups $P$ up to conjugacy. The virtual set of regular semisimple elliptic elements is given by

$$
\bigcap_{P}\left\{X \in \mathfrak{g}^{\text {reg,ss }}: \text { there does not exist } g \in G \text { such that } \operatorname{Ad} g(X) \in P\right\} \text {. }
$$

Example 6. Consider the virtual set in $G L(2)$ :

$$
Z=\left\{\left(\begin{array}{cc}
a & b \\
u b & a
\end{array}\right): \operatorname{ord}(u)=0 \wedge \operatorname{ord}(a) \geq 0 \wedge \operatorname{ord}(b) \geq 0 \wedge a^{2}-u b^{2} \neq 0 \wedge b \neq 0\right\} .
$$


This is a virtual subset of the set of regular semisimple elements. The condition for ellipticity for $X \in Z$ is

$$
\nexists A . A X-X A \in\left(\begin{array}{ll}
* & * \\
0 & *
\end{array}\right) \wedge(\operatorname{det} A \neq 0) .
$$

This is equivalent (excluding the prime 2) to the virtual subset of $Z$ given by the condition

$$
\nexists \xi \cdot \operatorname{ac}(u)=\xi^{2} .
$$

\section{An integration Formula}

Let $G=\bigsqcup_{t} K t K$ be the Cartan decomposition of the set $G$ of $p$-adic points of a split reductive group over a $p$-adic field. Assume $t \in T$, the set of points of a split torus. The method of Cartier gives the following integration formula for functions supported on a single double coset $K t K$.

Theorem 7. Let dk be a Haar measure on $K$. Let $d g$ be a Haar measure on $G$. Then there exists a constant $c(K t K)$ such that for all $f \in C_{c}(K t K)$,

$$
c(K t K) \int_{K \times K} f\left(k_{1} t k_{2}\right) d k_{1} d k_{2}=\int_{G} f(g) d g .
$$

Proof. Follow Cartier [2].

The constant can be obtained by taking $f$ to be the characteristic function of $K t K$. We find that

$$
c(K t K) \operatorname{vol}(K, d k)^{2}=\operatorname{vol}(K t K, d g) .
$$

Pick $d g$ so that its restriction to $K$ is $d k$, and normalize $d k$ so that

$$
\operatorname{vol}(K, d k)=\left|G\left(\mathbb{F}_{q}\right)\right| q^{-\operatorname{dim}(G)} .
$$

Let $[G]_{q}$ be the constant defined by the right-hand side of this equation.

\section{THE MAIN RESULT}

Let $\mathfrak{g}$ be a split reductive group over $\mathbb{Q}$. Let $G$ be a split reductive group over $\mathbb{Q}$ with Lie algebra $\mathfrak{g}$. We take $\mathfrak{g}$ and $G$ to be represented explicitly as matrices in $\mathfrak{g} l(n)$ and $G L(n)$, respectively. Let $O_{v}$ be the ring of integers of a completion $L_{v}$ of a number field $L / \mathbb{Q}$. We may assume that $G\left(O_{v}\right)=G L\left(n, O_{v}\right) \cap G\left(L_{v}\right)$ is a hyperspecial maximal compact subgroup for almost all places $v$ of $L$.

View $\mathfrak{g}$ and $G$ as virtual subsets of the virtual sets $\mathfrak{g} l(n)$ and $G L(n)$. Let $\mathfrak{g l}(n, O)$ be the virtual subset of $\mathfrak{g l}(n)$ given by

$$
\left\{\left(x_{i j}\right): \operatorname{ord}\left(x_{i j}\right) \geq 0, \forall i, j\right\} .
$$

Set $\mathfrak{g}(O)=\mathfrak{g} \mathbf{l}(n, O) \cap \mathfrak{g}$.

Let $f$ be a locally constant virtual function on $\mathfrak{g}$. Let $E$ be a virtual set of regular semisimple elliptic elements whose characteristic function is locally constant.

If $L$ is a finite extension of $\mathbb{Q}$, and $v$ is a place of $L$, the completion $L_{v}$ at $v$ is (the domain of) a structure for Pas's language. Let $O_{v}$ be the ring of integers of $L_{v}$. We write $f_{v}$ for the locally constant function in that structure. We write $E_{v}$ for the definable subset of $\mathfrak{g}\left(O_{v}\right)$ obtained from the virtual set $E$. Assume that for almost all $L_{v}, E_{v}$ is a compact set. (By 'almost all' here and in what follows, we 
mean all but finitely many residue field characteristics for $L_{v}$. The excluded set of primes is not allowed to depend on the number field $L$.)

We fix measures on $G_{v}$ (the set of $p$-adic points of $G$ ), and $\mathfrak{g}_{v}$ (the set of $p$-adic points of $\mathfrak{g}$ ) by fixing differential forms of top degree defined over $\mathbb{Q}$ on $G$ and $\mathfrak{g}$. Normalize $d X$ so that $\operatorname{vol}\left(\mathfrak{g}\left(O_{v}\right)\right)=1$. Normalize $d g$ to restrict to $d k$ on $G\left(O_{v}\right)$. Normalize $d k$ as in Section 4

Theorem 8. For each $f$ and $E$, there is a virtual motive

$$
M(f, E) \in K_{0}^{m o t}\left(\operatorname{Var}_{\mathbb{Q}}\right)\left[\mathbb{L}^{-1}\right] \otimes \mathbb{Q}
$$

and a finite set $S$ of primes with the following property: If $L$ is a finite extension of $\mathbb{Q}$ and $v$ is a place of $L$ that does not lie over any $p \in S$, then

$$
\operatorname{vol}(K, d k) \int_{E_{v}} \int_{G_{v}} f_{v}\left(g^{-1} X_{v} g\right) d g d X=\operatorname{trace}\left(\operatorname{Frob}_{v}, M(f, E)\right) .
$$

Remark 9. In general, a single elliptic element $X$ in a $p$-adic field is not definable and the conjugacy class $O(X)$ is not definable. Thus, it is not reasonable to ask for a virtual motive to represent the integral, except when averaged over $E$.

Remark 10. The same conclusion holds if we take our structures to be $\mathbb{F}_{q}((t))$ instead of $L_{v}$. The object $M(f, E)$ is the same in both cases. Hence, we find as a corollary that the orbital integrals are "the same" in zero characteristic and positive characteristic.

Proof. In the course of the proof we increase the size of the finite set $S$ of primes several times without a change in notation.

For any given $L_{v}$, the integral over $G_{v}$ is a sum of integrals over $K t K$, as we run over all double cosets. For any given $X \in E_{v}$, there are only finitely many double cosets involved (because $X$ is elliptic). The collection of double cosets required is locally constant in $X$, so there is a neighborhood of $X$ on which the finite set of cosets is the same as for $X$. By compactness on $E_{v}$, there is a finite cover by such neighborhoods. It follows that there is a finite set of double cosets depending only on $E_{v}\left(\right.$ and $\left.f_{v}\right)$.

For simplicity, we may assume that $f_{v}$ is a characteristic function at $L_{v}$ of a virtual set $D$. Let $m=\left(m_{1}, \ldots, m_{\ell}\right)$ be a tuple of integers with $\ell$ equal to the reductive rank. Let $T$ be a split torus that gives at each $p$-adic place the split torus that appears in the Cartan decomposition. Let $\phi: \mathbb{G}_{m}^{\times \ell} \rightarrow T$ be an isomorphism (defined over $\mathbb{Q}$ ) between a product of multiplicative groups and the diagonal torus $T$. Extend ord to ord ${ }^{\ell}: T \rightarrow \mathbb{Z}^{\ell}$ by

$$
T \rightarrow \mathbb{G}_{m}^{\ell} \rightarrow{ }^{\text {ord }^{\ell}} \mathbb{Z}^{\ell}
$$

Let $\theta\left(m, k_{1}, k_{2}, X\right)$ be the formula in Pas's language given by

$$
\begin{aligned}
& \exists t . \quad X \in E \wedge \operatorname{Ad}\left(k_{1} t k_{2}\right) X \in D \\
& \wedge \operatorname{ord}_{v}(t)=m \\
& \wedge k_{1}, k_{2} \in G(O) .
\end{aligned}
$$

Being in Pas's language, the formula is independent of the structure $L_{v}$. Let $\theta(m)$ be the formula

$$
\exists k_{1} k_{2} X . \theta\left(m, k_{1}, k_{2}, X\right) .
$$

The formula $\theta(m)$ has a bounded number of solutions in each structure $L_{v}$. By Theorem[2] there is a finite set $C \subset \mathbb{Z}^{\ell}$ that contains the solutions in $m$ for almost all 
structures $L_{v}$. This implies that we can pick a finite collection of double cosets that works simultaneously for almost all structures $L_{v}$. By the Cartan decomposition, we may assume that $m_{1} \geq m_{2} \geq \cdots$ for $m=\left(m_{1}, \ldots, m_{\ell}\right) \in C$. Write $K_{m}$ for the double coset

$$
K \phi\left(\pi^{m}\right) K
$$

By the Cartier-style integration formula (Section 4), the integral of the theorem can be written as

$$
\sum_{m \in C}\left[K_{m}: K\right] \int_{K \times K \times E_{v}} \operatorname{char}\left(\theta\left(m, k_{1}, k_{2}, X\right)\right) d k_{1} d k_{2} d X
$$

In general, if $\phi$ is any formula in Pas's language with free variables $m, x_{1}, \ldots, x_{k}$, $\xi$ of the three different sorts, let $\sigma_{N} \phi$ be the "stabilized" formula given by

$$
\begin{gathered}
\sigma_{N} \phi \quad\left(m, x_{1}, \ldots, x_{k}, \xi\right)=\exists x_{1}^{\prime} \cdots x_{k}^{\prime} . \\
\left(\left(\operatorname{ord}\left(x_{1}^{\prime}-x_{1}\right) \geq N\right) \wedge \cdots \wedge\left(\operatorname{ord}\left(x_{k}^{\prime}-x_{k}\right) \geq N\right) \wedge\right. \\
\left.\phi\left(m, x_{1}^{\prime}, \ldots, x_{k}^{\prime}, \xi\right)\right) .
\end{gathered}
$$

By Lemma 11 (below), there exists $N$ so that for almost all $L_{v}, \theta$ and its stabilization $\sigma_{N} \theta$ determine the same definable subset of $G\left(O_{v}\right) \times G\left(O_{v}\right) \times \mathfrak{g}\left(O_{v}\right)$. This allows us to replace $\theta$ with $\sigma_{N} \theta$ in the integral of Formula (2). The definable subassignment $C$ determined by $\sigma_{N} \theta$ is a weakly stable subset of $\mathcal{L}(G \times G \times \mathfrak{g})$ in the sense of Remark [5.

By the main theorem of Denef and Loeser [3], for each $m$ in the finite sum of Formula (2), there is a virtual motive $M_{m}$ such that the trace of Frobenius at $v$ computes this volume. In their theorem, the $p$-adic volume is to be computed with respect to the Serre-Oesterlé measure on the group

$$
G\left(O_{v}\right) \times G\left(O_{v}\right) \times \mathfrak{g}\left(O_{v}\right) .
$$

This measure is invariant under analytic isomorphisms of the set. In particular, it is invariant under the group action of

$$
G\left(O_{v}\right) \times G\left(O_{v}\right) \times \mathfrak{g}\left(O_{v}\right)
$$

on itself. Hence it is a Haar measure.

To work out the normalization of the Haar measure, we note that the SerreOesterlé measure coincides with the counting measure modulo a uniformizer, scaled by $q^{-\operatorname{dim}(X)}$. Thus, the volume of this group is

$$
[G]_{q} \times[G]_{q} .
$$

For the normalization of $d k$ and $d X$ chosen in Section 4 the measure is also $[G]_{q} \times$ $[G]_{q}$. Hence the Serre-Oesterlé measure coincides with the chosen Haar measure on this group.

The constant $\left[K_{m}: K\right]$, the number of cosets, is a polynomial in $q$ with rational coefficients by MacDonald's formula [13, sec. 3.2]. This can be converted to a virtual motive by replacing $q$ with $\mathbb{L}$.

By summing over the finite set of $m \in C$, we obtain the result.

Lemma 11. For each $m$, there exists $N$ such that for almost all $L_{v}$, there is an equality of definable subsets of $G\left(O_{v}\right) \times G\left(O_{v}\right) \times \mathfrak{g}\left(O_{v}\right)$ :

$$
\left\{\left(k_{1}, k_{2}, X\right): \theta\left(m, k_{1}, k_{2}, X\right)\right\}=\left\{\left(k_{1}, k_{2}, X\right): \sigma_{N} \theta\left(m, k_{1}, k_{2}, X\right)\right\} .
$$


Proof. Fix $m$. For each structure $L_{v}$, it follows directly from compactness of $E_{v}$, $K_{m}$, and the local constancy of $D_{v}$ and $E_{v}$ that the corresponding $p$-adic set is a locally constant subset of $G\left(O_{v}\right) \times G\left(O_{v}\right) \times \mathfrak{g}\left(O_{v}\right)$. (This is an expression of the well-known fact that orbital integrals are locally constant on the set of regular semisimple elements.)

Extend ord to a function symbol on matrices $X=\left(x_{i j}\right)$ by defining

$$
\operatorname{ord}(X)=\min _{i j} \operatorname{ord}\left(x_{i j}\right) .
$$

Let $\psi_{\theta}(n)=\psi_{\theta m}(n)$ be the auxiliary formula in Pas's language given by

$$
\begin{aligned}
& \forall k_{1} k_{2} k_{1}^{\prime} k_{2}^{\prime} X X^{\prime} . \\
& \quad\left(\operatorname{ord}\left(k_{1}-k_{1}^{\prime}\right) \geq n \wedge \operatorname{ord}\left(k_{2}-k_{2}^{\prime}\right) \geq n \wedge \operatorname{ord}\left(X-X^{\prime}\right) \geq n\right) \Rightarrow \\
& \quad\left(\theta\left(m, k_{1}, k_{2}, X\right) \Leftrightarrow \theta\left(m, k_{1}^{\prime}, k_{2}^{\prime}, X^{\prime}\right)\right) .
\end{aligned}
$$

The formula asserts that the level is $n$. Let $\psi_{\theta}^{\prime}(n)$ be the formula in Pas's language given by

$$
\psi_{\theta}(n) \wedge\left(\forall n^{\prime}<n . \neg \psi \theta\left(n^{\prime}\right)\right) .
$$

It asserts that $n$ is the least level. By the local constancy of each structure $L_{v}$ and Theorem [2] we find that there is a level $n$ that works for almost all structures.

\section{EfFective Calculations}

Theorem 12. The virtual motive $M(f, E)$ is effectively computable from the data $f$ and $E$.

Remark 13. More precisely, we compute $M(f, E)$ as an explicit linear combination of classes $[X]$, where each $X$ is a smooth projective scheme over $\mathbb{Q}$. See [3] sec. 1.3]. Each scheme $X$ is presented through a finite number of coordinate patches. Each patch is given by an explicit list of polynomials $f_{1}, \ldots, f_{k}$ in $n$ variables such that the affine patch is isomorphic to $\mathbb{Q}\left[x_{1}, \ldots, x_{n}\right] /\left(f_{1}, \ldots, f_{k}\right)$. The maps between coordinate patches are given by explicit polynomials.

Proof. It is enough to prove the result when $f=\chi_{D}$, a locally constant virtual characteristic function.

Pas's algorithm is an effective procedure [14]. In fact, although Pas assumes for simplicity that the residue field has characteristic zero, the finite set of prime characteristics that must be avoided (for a given formula in the language) can be effectively calculated from his algorithm.

The Presburger algorithm is an effective procedure [8].

Combining Pas's algorithm and the Presburger algorithm, we have an effective procedure for determining effective bounds on the solutions of each $L_{i}$ of Formula (1), for each $i \in B$. Thus, the set $C$ is effectively computable. This means that an effective bound can be obtained on the number of double cosets that must be considered.

The natural number $N$ in Lemma 11 can be effectively computed for the same reason that $C$ can be. The subassignment attached to $\sigma_{N} \theta$ is weakly stable of level $N$. By quantifier elimination, for each $m$, the formula $\sigma_{N} \theta\left(m, k_{1}, k_{2}, X\right)$ can be replaced by an explicit special formula (in the sense of [3, sec. 5.3]). Truncation $\tau_{N}\left(\sigma_{N} \theta\right)$ at level $N$ gives a formula in $\mathcal{L}_{N}(G \times G \times \mathfrak{g})$ (the truncated arc-space of [3, sec. 5.4]), or equivalently the formula

$$
\theta^{\prime}=\tau_{N}\left(\sigma_{N} \theta\right) \wedge(x \in G(O) \times G(O) \times \mathfrak{g}(O)) .
$$


This formula may be considered as a formula in the first-order theory of rings. (More explicitly, we work in the structure $\mathbb{Q}[[t]]$. We replace quantification of $k_{1}$, $k_{2}, X$ with quantifiers over the matrix coefficients, and each matrix coefficient is expanded as a truncated power series $x_{i j}=z_{0}+z_{1} t+z_{2} t^{2}+\cdots$.)

By the definitions in [3], we have

$$
M(f, E)=\chi_{c}\left(\theta^{\prime}\right) \mathbb{L}^{-(n+1) d},
$$

where $d$ is the dimension of $G \times G \times \mathfrak{g}$.

To conclude the proof, we check that $\chi_{c}$ can be made effective for presented formulas $\theta^{\prime}$ in the first-order theory of rings. The procedure is as follows. We write $\theta^{\prime}$ in prenex normal form to get a Galois formula as in [3, sec. 2.2]. Quantifier elimination transforms the Galois formula into a Galois stratification. This procedure is effective [9, ch. 26]. (See also [3, 2,3,3].) The Galois group is presented as an explicit subgroup of a symmetric group. Part of the data obtained from the Galois stratification is a central function on the Galois group.

By [9, 26.12], Artin induction is effective, giving the central function as an explicit rational combination of characters induced from the trivial character on cyclic subgroups.

By refining the Galois stratification as necessary, we may assume that the Galois stratification is affine, that is, each $C / A$ is a ring cover in the sense of [9]. Artin induction and the properties of $\chi_{c}$ established in [3] reduce the problem to computing $\chi_{c}(X / H)$, where $X$ is affine with a cyclic group action $H$, and the data $(X, H)$ are explicitly given. The calculation of the quotient amounts to computing the invariants of $\mathbb{Q}[X]$ under the finite group $H$. There are several implementations of algorithms to compute this ring of invariants. (A survey of implementations appears for example in [6, page 2].)

To compute the class $\chi_{c}(X)$ where $X$ is an affine variety, embed it in a smooth projective variety $\tilde{X}$. By constructive resolution of singularity algorithms ([1] and [7]), $\tilde{X}$ can be effectively computed from $X$. By the properties of the map $\chi_{c}$,

$$
\chi_{c}(X)=[\tilde{X}]-\chi_{c}(D) .
$$

The divisor $D$ has lower dimension. Part of the constructive resolution of singularities gives a description of the irreducible components of $D$. By induction on dimension, we may assume that $\chi_{c}(D)$ is known.

This completes the proof that $M(f, E)$ can be effectively computed.

\section{REFERENCES}

[1] G. Bodnár and J. Schicho, A computer program for the resolution of singularities, in Resolution of singularities (Obergurgl, 1997), Progr. Math., 181, Birkhäuser, Basel, 2000, pp. 231-238. MR1748621 (2001e:14001)

[2] P. Cartier, Representations of $p$-adic groups: a survey, in Automorphic Forms, Representations, and $L$-functions, Corvallis, Symp. in Pure Math, AMS XXXIII, part 1, 1977. MR.0546593(81e:22029)

[3] J. Denef, F. Loeser, Definable sets, motives, and p-adic integrals, Journal of the AMS, 14, No. 2, 429-469 (2001). MR1815218 (2002k:14033)

[4] J. Denef, F. Loeser, Motivic Integration and the Grothendieck Group of Pseudo-Finite Fields, Proc. of the Int. Congress of Math. 2002. Vol II, 13-23, Higher Ed. Press, Beijing. MR 1957016 (2004f:14040)

[5] J. Denef, F. Loeser, One some rational generating series occurring in arithmetic geometry, preprint math.NT/0212202. 
[6] H. Derksen, Constructive Invariant Theory and the Linearization Problem, Ph.D. thesis, University of Basel, 1997, http://www.math.lsa.umich.edu/ hderksen/preprint.html.

[7] S. Encinas and H. Hauser, Strong resolution of singularities in characteristic zero, Comment. Math. Helv. 77 (2002), no. 4, 821-845. MR1949115 (2004c:14021)

[8] H. B. Enderton, A mathematical introduction to logic. Second edition. Harcourt/Academic Press, Burlington, MA, 2001. MR1801397 (2001h:03001)

[9] M. D. Fried, M. Jarden, Field arithmetic. Ergebnisse der Mathematik und ihrer Grenzgebiete (3) [Results in Mathematics and Related Areas (3)], 11. Springer-Verlag, Berlin, 1986. MR:0868860 (89b:12010)

[10] J. Gordon, thesis, University of Michigan, 2002.

[11] J. Gordon and T. Hales, Virtual Transfer Factors, Represent. Theory 7 (2003), 81-100. (preprint http://xxx.lanl.gov/abs/math.RT/0209001). MR1973368 (2004c:11084)

[12] T. C. Hales. Can p-adic integrals be computed? to appear, http://xxx.lanl.gov/abs/ math.RT/0205207.

[13] I. G. MacDonald, Spherical Functions on a Group of $p$-adic Type, Publ. of the Ramanujan Inst. Number 2, 1971. MR0435301 (55:8261)

[14] J. Pas, Uniform p-adic cell decomposition and local zeta functions. J. Reine Angew. Math. 399 (1989), 137-172. MR/1004136|(91g:11142)

Department of Mathematics, University of Pittsburgh, Pittsburgh, Pennsylvania 15260

E-mail address: hales@pitt.edu 